\title{
Desarrollo de un Método Micropotenciométrico de Bajo Costo para la Determinación de Acidez Valorable
}

\author{
WILLIAM CALERO CÁCERESa*, PABLO BONILLAa \\ a Laboratorio de Coloideoquímica, Facultad de Ciencias Químicas, Universidad Central del Ecuador, Quito. \\ *Correspondencia: wcaleroc@hotmail.com \\ Recibido: 22 enero 2011 / Aceptado: 17 septiembre 2011
}

\section{Resumen}

Se desarrolló un método micropotenciométrico de bajo costo para la determinación de acidez valorable en muestras alimentarias, matriz jugos, utilizando materiales de fácil adquisición para su construcción, como son sueldas de tungsteno puro de $1.6 \mathrm{~mm} \times 175 \mathrm{~mm}$, alambres de cobre de uso eléctrico de $0.1 \mathrm{~mm}$, multímetro genérico, puntas de pipeta plásticas, algodón y silicona. Con la finalidad de mantener la rigidez analítica el método fue optimizado y evaluado estadísticamente por medio de ensayos de linealidad, exactitud, precisión, estabilidad, límite de detección y cuantificación utilizando ácido cítrico como analito diana. Con el nuevo método se realizó un estudio comparativo frente al método potenciométrico clásico con electrodo de vidrio por medio del análisis de la recta de regresión y el estadístico t como confirmación, demostrando su aplicabilidad para el análisis de muestras alimenticias, obteniéndose una alternativa con ventajas analíticas, económicas y ambientales.

Palabras clave: Análisis de alimentos, Análisis Electroquímico, Química Analítica Instrumental, Jugos de frutas, Bebidas refrescantes.

\section{Development of a Low-Cost Micropotentiometric Method for the Determination of Titratable Acidity}

\begin{abstract}
We developed a low-cost micropotentiometric method for determining titratable acidity in food samples, matrix juices, using readily available materials for construction, such as pure tungsten welds of $1.6 \mathrm{~mm} \times 175 \mathrm{~mm}$, copper wire of $0.1 \mathrm{~mm}$ for electrical use, generic multimeter, plastic pipette tips, cotton and silicone.

In order to maintain the analytical rigidity, the method was optimized and evaluated statistically by tests of linearity, accuracy, precision, stability, limit of detection and quantification using citric acid as a target analyte.

With the new method, the results were evaluated according to the comparative study against the classical potentiometric method with glass electrode through the analysis of the regression line and the statistic $t$ as confirmation, demonstrating its applicability for the analysis of food samples.
\end{abstract}

Keywords: food Analysis, Electrochemical Analysis, Instrumental Analytical Chemistry, Fruit juices, Soft drinks. 


\section{Introducción}

Para determinar acidez valorable, se titula un volumen (o un peso) conocido de una muestra alimentaria frente a una base valorada, bien sea hasta un punto final señalado por el pH por un método potenciométrico o bien hasta el de la fenolftaleína [1]. El punto final electroquímico es ampliamente aplicado y proporciona datos inherentemente más exactos que el método correspondiente que utiliza indicadores [2].

Las titulaciones ácido-base con monitoreo potenciométrico para análisis químico o estudios de procesos en solución se ve limitada por el alto costo de los sensores de $\mathrm{pH}$ a base de membrana de vidrio combinados con electrodos de calomel saturado. Además, el tamaño de estos electrodos exige la utilización de celdas de reacción y buretas de gran volumen.

Si bien el electrodo de vidrio es muy eficiente en soluciones acuosas diluidas, no es posible miniaturizar membranas de vidrio y los ultramicroelectrodos de $\mathrm{pH}$ a base de intercambiadores líquidos tienen un costo muy elevado [3]. Además, los electrodos de vidrio poseen naturaleza frágil y características especiales que limitan sus aplicaciones in-situ [4]. Por estos motivos se plantea buscar una nueva alternativa a las titulaciones potenciométricas clásicas, que ofrezca resultados validados, de bajo costo y de aplicación sencilla.

Los óxidos metálicos han sido utilizados en el diseño de electrodos electroquímicos en forma de películas o estructuras [5]. Las superficies metálicas que forman los hidróxidos insolubles en soluciones acuosas pueden ser utilizadas para determinaciones de $\mathrm{pH}$ como el estaño, plomo, arsénico, antimonio, bismuto, tungsteno, cobre, plata, oro, zinc, mercurio, tantalio, renio, hierro, osmio, rodio, iridio y platino [6-9]. Podemos desplazar el potencial electroquímico de una celda cambiando la concentración de una especie no electroactiva presente en el equilibrio electroquímico, aplicado al sistema tungsteno/óxido de tungsteno:

$$
\begin{gathered}
W+2 \mathrm{H}_{2} \mathrm{O} \leftrightarrow W \mathrm{O}_{2}+4 H^{+}+4 e^{-} \\
E=E^{0}+\frac{2.303 R T}{4 F} \log \frac{\left[W \mathrm{O}_{2}\right]\left[\mathrm{H}^{+}\right]^{4}}{[W]\left[\mathrm{H}_{2} \mathrm{O}\right]^{2}} \\
E=E^{0}+\frac{2.303 R T}{F} p H
\end{gathered}
$$

También se han obtenido polímeros como excelentes sensores de $\mathrm{pH}$ [10-12].
El tungsteno ha sido estudiado como sensor de $\mathrm{pH}$ desde mediados del siglo pasado en la investigación de $\mathrm{pH}$ intracelular [13], también en prototipos de sensores de $\mathrm{pH}$ para potenciómetros de inyección de flujo a microescala aplicado al análisis de bebidas alcohólicas y de muestras de agua ambiental [14]. Baeza y Vargas et al. han efectuado estudios utilizando un microelectrodo sensor a base de $\mathrm{W}^{0}$, un microelectrodo de referencia de $\mathrm{Cu}|\mathrm{Cu}(\mathrm{II})| \mathrm{H}_{2} \mathrm{O} \mid$ y $\mathrm{Cu}|\mathrm{Cu}(\mathrm{II})| \mathrm{CuSO}_{4}||$ y la utilización de materiales de bajo costo, para la fabricación de material volumétrico como la microbureta a microescala total integrada en una celda para titulaciones ácido-base, a la par se reportan estudios comparativos obteniéndose resultados similares en la titulación de ácidos minerales monopróticos y de sales ácidas de potasio, en comparación con el electrodo de vidrio y calomel saturado [15-16].

Por lo que, en base a los antecedentes relacionados, en este trabajo se planteó desarrollar y evaluar la factibilidad de un método con sensores de $\mathrm{pH}$ y de referencia de bajo costo para la valoración potenciométrica de la acidez valorable, utilizando materiales de fácil adquisición, como una alternativa al método clásico con electrodo indicador de vidrio.

\section{Materiales y Métodos}

\subsection{Reactivos}

Hidróxido de Sodio p.a., Ftalato Ácido de Potasio p.a. Sulfato cúprico p.a. y Ácido Cítrico p.a. adquiridos en Merck (Darmstadt, Alemania), Soluciones tampón de $\mathrm{pH} 4.01,7.00$ y 9.21 adquiridos en Mettler Toledo (Gieben, Alemania).

\subsection{Soluciones y muestras}

Se preparó una solución de Hidróxido de Sodio $(\mathrm{NaOH}) 0.1 \mathrm{~N}$ aproximadamente valorándola frente a un estándar de Ftalato Ácido de Potasio (KHF), para el estudio estadístico se prepararon soluciones estándar de ácido cítrico en un rango entre $0.06 \pm 0.01 \%(p / v)$ y $10.18 \pm 0.11 \%(p / v)$, y para el estudio comparativo se utilizaron 30 muestras de jugos en sus variedades natural, bebidas tipo jugo, néctares y zumos. 


\subsection{Construcción de electrodos}

Para el electrodo indicador se utilizó una barra de tungsteno (pureza $>99.5 \%$ ) para soldadura adquirida en Weldcraft (Appleton, EUA), se corta un segmento de $3.830 \pm 0.005 \mathrm{~cm}$ de largo y $2.310 \pm 0.005$ $\mathrm{cm}$ de ancho (fijo de la barra), se introduce en un tip de plástico (utilizados en las pipetas automáticas) de $5.000 \times 0.815 \mathrm{~cm}$, y se sujeta con silicón. Para el electrodo de referencia se utilizó un alambre de cobre de 14", utilizados en sistemas eléctricos, se cortan dos segmentos de $4.750 \pm 0.005 \mathrm{~cm}$ de largo y 0.145 $\pm 0.005 \mathrm{~cm}$ de ancho (fijo del alambre), los cuales son introducidos en dos tips de plástico de $5.000 \times$ $0.815 \mathrm{~cm}$, y se fija con silicona. Finalmente, se embona en otros tips de plástico de $3.635 \times 0.700 \mathrm{~cm}$, los cuales tienen en su parte final un pedazo de algodón compacto que sirve para aislar la solución interna del microelectrodo de referencia, que fue agua destilada descarbonatada y sulfato de cobre $1 M$; se procuró mantener constante la distancia entre el pedazo de algodón y el alambre de cobre, encontrándose en un rango de $0.240 \pm 0.010 \mathrm{~cm}$.

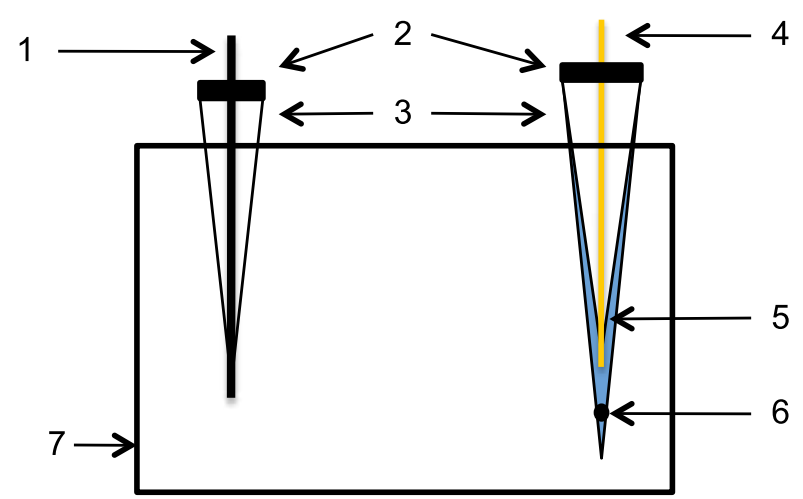

Figura 1. Microcelda potenciométrica de bajo costo para la determinación de pH. 1: Barra de tungsteno. 2: Silicona en barra. 3: Tips de pipeta automática. 4: Alambre de cobre $4.5 \mathrm{~cm} \times 0.1 \mathrm{~cm}$. 5: Solución de $\mathrm{CuSO}_{4} 1 \mathrm{M}$ o agua destilada descarbonatada. 6: Algodón. 7: Vaso de $15 \mathrm{~cm}^{3}$.

\subsection{Método micropotenciométrico de bajo costo para la determinación de acidez valorable}

Se analizó el desempeño de dos sistemas electroquímicos:

Sistema 1: $(+) \mathrm{Cu}\left|\mathrm{Cu}^{2+}\right| \mathrm{H}_{2} \mathrm{O}|| \mathrm{W}^{0}$

Sistema 2: $(-) \mathrm{Cu}\left|\mathrm{Cu}^{2+}\right| \mathrm{Cu}_{2} \mathrm{SO}_{4}, 1 \mathrm{M}|| \mathrm{W}^{0}$
Y la cantidad de muestra $[0.50 \mathrm{~mL}(+)$ y $1.00 \mathrm{~mL}(-)]$ frente a una muestra comercial de concentración desconocida, obteniéndose la siguiente matriz de experimentos:

Tabla 1. Matriz de experimentos para la selección del sistema electroquímico

\begin{tabular}{ccccc}
\hline Exp. & Cod. & $\begin{array}{c}\text { Sistema } \\
\text { electroquímico } \\
\text { (A) }\end{array}$ & $\begin{array}{c}\text { Cantidad } \\
\text { muestra } \\
\text { (B) }\end{array}$ & $\begin{array}{c}\text { Interacción } \\
\text { AB }\end{array}$ \\
\hline 1 & 1 & - & - & + \\
2 & $\mathrm{a}$ & + & - & - \\
3 & $\mathrm{~b}$ & - & + & - \\
4 & $\mathrm{ab}$ & + & + & + \\
\hline
\end{tabular}

Se añade con una pipeta la muestra de jugo a un vaso plástico de bajo costo de $15 \mathrm{~mL}$ de capacidad, con agua destilada descarbonatada se completa un volumen de $3 \mathrm{~mL}$, se coloca un mini magneto y se tapa el vaso. Se insertan los electrodos en orificios hechos en la tapa del vaso, se acopla en el agitador magnético y a la microbureta rellenada de $\mathrm{NaOH}$ $0.1 \mathrm{~N}$, se utiliza un multímetro de bajo costo para la medición de la diferencia de potencial entre el electrodo de $\mathrm{W}$ y el electrodo de referencia de Cu/ $\mathrm{Cu}^{2+}$, el microelectrodo de referencia se conecta a la entrada de tierra del voltímetro. Mientras se añade la base titulante se registran los datos de potencial eléctrico. Se efectuó el análisis por quintuplicado y los parámetros indicadores fueron el análisis de varianza [17] acoplado al diseño factorial $2^{k}$, la desviación estándar, el rango y el coeficiente de variación.

Con la finalidad de evaluar el comportamiento lineal de los sistemas electroquímicos 1 y 2 , se construyeron curvas de calibración con soluciones tampón de $\mathrm{pH}$ 4.01 , $\mathrm{pH} 7.00$ y pH 9.21, rango que cubre el pH en una valoración ácido-base, por cuatro días con tres repeticiones. Como indicador se utilizó la ecuación de Nikol'skii que relaciona el potencial eléctrico medido en función del $\mathrm{pH}$, contrastando la pendiente y el coeficiente de correlación mediante el análisis de la † de comparación de medias [18].

Además, ambos sistemas fueron sometidos a un control de estabilidad en función del tiempo utilizándose (4) soluciones tampón, como indicador se tuvo el rango (5) de dispersión y el tiempo de respuesta. 


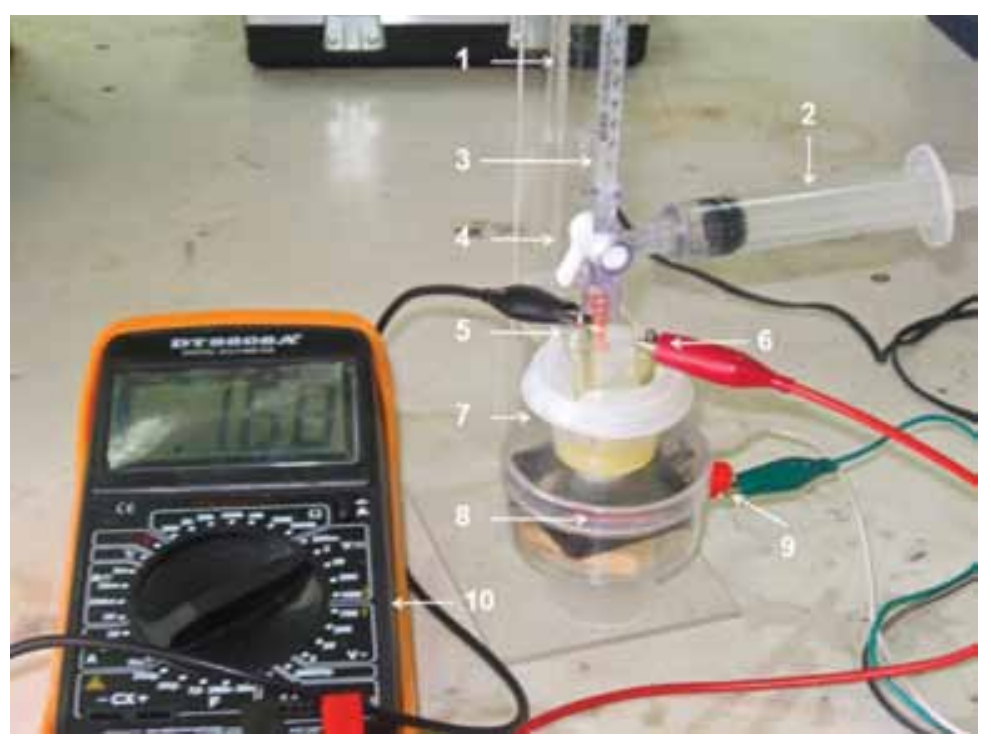

Figura 2. Sistema electroquímico de bajo costo. 1: Soporte de acrílico. 2: Jeringa dispensadora de solución titulante. 3: Jeringa de $1 \mathrm{~mL}$ con división de $0.01 \mathrm{~mL}$. 4: Llave clínica de 3 vías. 5: Electrodo de referencia de $\mathrm{Cu} / \mathrm{Cu}^{2+}$. 6: Electrodo indicador de W. 7: Vaso de $15 \mathrm{~mL}$ con muestra. 8: Ventilador de computador acoplado con una resistencia de 130 W y un imán. 9: Entrada para fuente de 9V. 10: Multímetro de bajo costo.

\subsection{Estudio estadístico}

Se determinó el límite de detección (LD) y el límite de cuantificación (LC) del método micropotenciométrico de bajo costo por medio del análisis de 20 muestras blanco, se consideró la media de los resultados más tres desviaciones estándar en el caso del LD y seis desviaciones estándar en el caso del LC [19-20].

Para el parámetro de exactitud y precisión, se analizaron soluciones a cinco niveles de concentración de ácido cítrico entre $0.06 \%(\mathrm{p} / \mathrm{v})$ y $10.18 \%(\mathrm{p} / \mathrm{v})$ por medio del método micropotenciométrico de bajo costo durante tres días con diez repeticiones por nivel; para la precisión el parámetro indicador fue el Análisis de Varianza de dos factores, para la exactitud se evaluaron la desviación estándar, el coeficiente de variación el error absoluto y relativo.

Se analizó la sensibilidad del método por medio de cinco pequeños incrementos de concentración de ácido cítrico, el parámetro indicador fue el porcentaje de recuperación.

\subsection{Método potenciométrico utilizando electrodo de vidrio}

Se utilizó una variación del método AOAC 942.15 el cual establece que el punto final por medición potenciométrica es el volumen de titulante necesario para al- canzar un pH de 8.2, como en este estudio se procuró evaluar el comportamiento del sistema potenciométrico medido en actividades electroquímicas, se tomará como punto final los obtenidos mediante métodos gráficos.

Se transfiere con una pipeta volumétrica $10 \mathrm{~mL}$ de la muestra en un vaso de precipitación de $100 \mathrm{~mL}$, se añade, aproximadamente $20 \mathrm{~mL}$ de agua destilada descarbonatada, se coloca un magneto y se acopla el vaso en un agitador magnético, se inserta el electrodo de vidrio de un potenciómetro comercial dentro de la solución problema. Mediante una bureta de $10 \mathrm{~mL}$ rellenada de $\mathrm{NaOH}$ aproximadamente $0.1 \mathrm{~N}$, se añade la base titulante, a la par se registran las medidas de potencial eléctrico. En el presente estudio se utilizó el potenciómetro WTW Inolab 720.

\subsection{Estudio comparativo}

Se analizaron 30 muestras de jugos y bebidas en un amplio intervalo de concentraciones por medio del método potenciométrico con electrodo de vidrio (referencia) y el método micropotenciométrico de bajo costo por triplicado. Como parámetros indicadores se tuvieron a la recta de regresión, pendiente, ordenada y el coeficiente de correlación momento-producto. Como complemento se utiliza el contraste " $t$ " para datos emparejados con la finalidad de evaluar si existe diferencia significativa entre los resultados obtenidos para cada análisis. 


\section{Resultados y Discusión}

\subsection{Desarrollo y optimización del método}

Los resultados de la matriz de experimentos 1 se detallan a continuación:

Tabla 2. Resultados del análisis de acidez valorable de una muestra de limonada de acuerdo al diseño factorial $2^{2}$.

\begin{tabular}{ccccccc}
\hline Exp. & Cod. & \multicolumn{5}{c}{ Acidez Valorable } \\
& & expresada como \% ácido cítrico $(\mathbf{p} / \mathbf{v})$ \\
\cline { 3 - 7 } & $\mathbf{y}_{1}$ & $\mathbf{y}_{2}$ & $\mathbf{y}_{3}$ & $\mathbf{y}_{4}$ & $\mathbf{y}_{5}$ \\
\hline 1 & 1 & 0,27 & 0,26 & 0,27 & 0,26 & 0,27 \\
2 & a & 0,26 & 0,27 & 0,27 & 0,26 & 0,26 \\
3 & $\mathrm{~b}$ & 0,28 & 0,27 & 0,30 & 0,28 & 0,27 \\
4 & ab & 0,26 & 0,28 & 0,27 & 0,26 & 0,27 \\
\hline
\end{tabular}

Tabla 3. Parámetros de dispersión para la optimización de electrodos.

\begin{tabular}{ccccc}
\hline Exp. & Cod. & $\hat{\mathbf{y}}(\%$ AC) & S (\% AC) & CV (\%) \\
\hline 1 & 1 & 0,27 & 0,01 & 2,06 \\
2 & $\mathrm{a}$ & 0,26 & 0,01 & 2,07 \\
3 & $\mathrm{~b}$ & 0,28 & 0,01 & 4,37 \\
4 & $\mathrm{ab}$ & 0,27 & 0,01 & 3,12 \\
\hline
\end{tabular}

Tabla 4. Análisis de Varianza $2^{k}$ para la selección de electrodos.

\begin{tabular}{ccccccc}
\hline $\begin{array}{c}\text { Fuente de } \\
\text { variación }\end{array}$ & $\begin{array}{c}\text { Suma de } \\
\text { cuadrados }\end{array}$ & $\begin{array}{c}\text { Cuadrado } \\
\text { medio }\end{array}$ & GL & F & $\begin{array}{c}\text { F tab } \\
\mathbf{9 5 \%}\end{array}$ & $\begin{array}{c}\mathbf{F} \text { tab } \\
\mathbf{9 9 \%}\end{array}$ \\
\hline A & $2,45 \mathrm{E}-04$ & $2,45 \mathrm{E}-04$ & 1 & $3,48^{\text {ns }}$ & 4.49 & 8.53 \\
B & $4,05 \mathrm{E}-04$ & $4,05 \mathrm{E}-04$ & 1 & $5,76^{*}$ & & \\
AB & $1,25 \mathrm{E}-04$ & $1,25 \mathrm{E}-04$ & 1 & $1,78^{\text {ns }}$ & & \\
Error & $1,13 \mathrm{E}-03$ & $7,03 \mathrm{E}-05$ & 16 & & & \\
Total & $1,90 \mathrm{E}-03$ & & 19 & & & \\
\hline
\end{tabular}

No existe significancia en la obtención de resultados entre el sistema electroquímico con electrodo de referencia de $\mathrm{Cu}\left|\mathrm{Cu}^{2+}\right| \mathrm{H}_{2} \mathrm{O}|| \mathrm{W}^{0}$ y su homólogo de $\mathrm{Cu}\left|\mathrm{Cu}^{2+}\right| \mathrm{Cu}_{2} \mathrm{SO}_{4}, 1 \mathrm{M}|| \mathrm{W}^{0}$ al $95 \%$ y $99 \%$ de confianza, la fuente de variación de la cantidad de mues- tra es significativa al 95\% de confianza, la interacción entre ambos efectos, no es significativa al $95 \%$ y $99 \%$ de confianza. Con respecto al análisis de las gráficas obtenidas en la valoración micropotenciométrica para ambos sistemas, en las representaciones $\mathrm{eV}$ vs. $\mathrm{V} \mathrm{NaOH}$ se observa que el sistema 1 posee una rampa de decrecimiento de potencial eléctrico con una pendiente más pronunciada que el sistema 2, lo cual influye de manera directa en la obtención de los puntos finales de titulación.

Con respecto a la cantidad de muestra, se observa un aumento del coeficiente de variación de manera inversamente proporcional a la cantidad de muestra, en especial para la celda electroquímica con sulfato de cobre $1 \mathrm{M}$ como solución interna en el electrodo de referencia, por lo que se decidió utilizar $1.00 \mathrm{~mL}$ de muestra, cantidad con la cual se obtienen coeficientes de variación razonablemente bajos $(\mathrm{CV} \approx 2 \%)$.

Con respecto al comportamiento lineal de los sistemas electroquímicos frente al $\mathrm{pH}$ se obtuvieron los siguientes resultados:

Tabla 5. Resultados de la medición del potencial eléctrico (V) en función del pH para el sistema electroquímico 1 $|\mathrm{Cu}| \mathrm{Cu}^{2+}\left|\mathrm{H}_{2} \mathrm{O}\right| \mid W \%$.

\begin{tabular}{|c|c|c|c|c|c|c|}
\hline \multirow[t]{2}{*}{ Día } & \multirow[t]{2}{*}{$\mathrm{pH}$} & \multicolumn{3}{|c|}{ Mediciones (V) } & \multirow{2}{*}{$\begin{array}{l}\text { Media } \\
\text { (V) }\end{array}$} & \multirow{2}{*}{$\begin{array}{l}\mathbf{s} \\
(\mathrm{V})\end{array}$} \\
\hline & & 1 & 2 & 3 & & \\
\hline \multirow{3}{*}{1} & 4,01 & $-0,079$ & $-0,080$ & $-0,078$ & $-0,079$ & 0,001 \\
\hline & 7,00 & $-0,211$ & $-0,210$ & $-0,211$ & $-0,211$ & 0,001 \\
\hline & 9,21 & $-0,326$ & $-0,328$ & $-0,320$ & $-0,325$ & 0,004 \\
\hline \multirow{3}{*}{2} & 4,01 & $-0,073$ & $-0,073$ & $-0,072$ & $-0,073$ & 0,001 \\
\hline & 7,00 & $-0,216$ & $-0,215$ & $-0,217$ & $-0,216$ & 0,001 \\
\hline & 9,21 & $-0,328$ & $-0,327$ & $-0,328$ & $-0,328$ & 0,001 \\
\hline \multirow{3}{*}{3} & 4,01 & $-0,067$ & $-0,065$ & $-0,065$ & $-0,066$ & 0,001 \\
\hline & 7,00 & $-0,188$ & $-0,188$ & $-0,189$ & $-0,188$ & 0,001 \\
\hline & 9,21 & $-0,317$ & $-0,315$ & $-0,312$ & $-0,315$ & 0,003 \\
\hline \multirow{3}{*}{4} & 4,01 & $-0,118$ & $-0,116$ & $-0,115$ & $-0,116$ & 0,002 \\
\hline & 7,00 & $-0,216$ & $-0,214$ & $-0,215$ & $-0,215$ & 0,001 \\
\hline & 9,21 & $-0,330$ & $-0,329$ & $-0,330$ & $-0,330$ & 0,001 \\
\hline
\end{tabular}


A

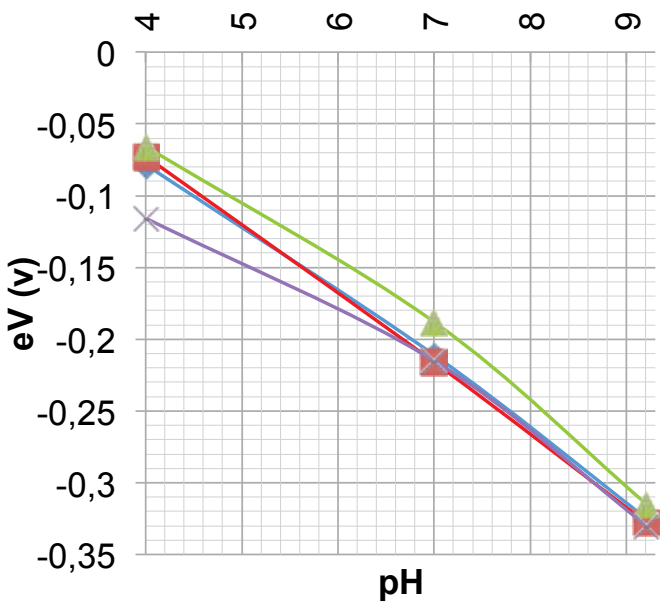

B

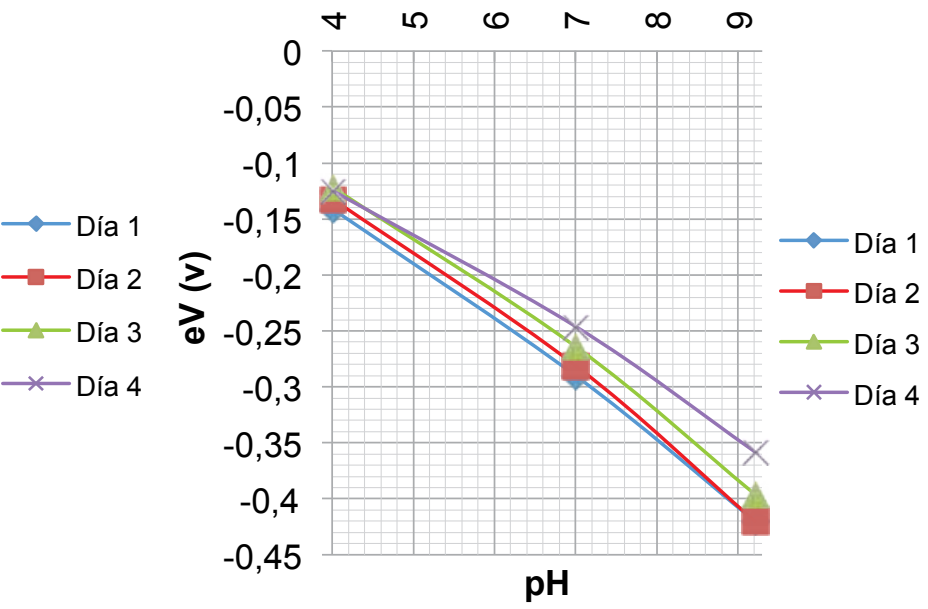

Figura 3. Ecuaciones de Nikol'skii, A: Sistema electroquímico 1, B: Sistema electroquímico 2.

Tabla 6. Sensibilidad de calibración, coeficientes de correlación y contraste t de sensibilidad. Ecuación de Nikol'skii para los sistemas electroquímicos 1 y 2 .

\begin{tabular}{ccccc|ccc}
\hline & \multicolumn{2}{c}{ Sistema 1 } & \multicolumn{2}{c|}{ Sistema 2 } & \multicolumn{3}{c}{$\begin{array}{c}\text { Contraste "t" } \\
\text { Sensibilidad }\end{array}$} \\
\cline { 2 - 8 } & $\begin{array}{c}\mathbf{m} \\
\left(\mathbf{V} \cdot \mathbf{p H}^{-1}\right)\end{array}$ & $\mathbf{r}$ & $\begin{array}{c}\mathbf{m} \\
\left(\mathbf{V} \cdot \mathbf{p H}^{-1}\right)\end{array}$ & $\mathbf{r}$ & $\mathbf{t}$ & $\begin{array}{c}\mathbf{t} \text { crítico } \\
\mathbf{9 5 \%}\end{array}$ & $\begin{array}{c}\mathbf{t} \text { crítico } \\
\mathbf{9 9 \%}\end{array}$ \\
\hline Día 1 & $-0,047$ & 0,998 & $-0,053$ & 0,997 & $1,683^{\text {ns }}$ & 2,447 & 3,707 \\
Día 2 & $-0,049$ & 0,999 & $-0,055$ & 0,995 & & & \\
Día 3 & $-0,047$ & 0,991 & $-0,052$ & 0,995 & & & \\
Día 4 & $-0,040$ & 0,983 & $-0,044$ & 0,996 & & & \\
Media & $-0,046$ & 0,993 & $-0,051$ & 0,996 & & & \\
Desv. Est. & 0,004 & 0,007 & 0,005 & 0,001 & & & \\
\hline
\end{tabular}

No existe significancia en la sensibilidad de calibración entre el sistema electroquímico con electrodo de referencia de $\mathrm{Cu}\left|\mathrm{Cu}^{2+}\right| \mathrm{H}_{2} \mathrm{O}|| W^{0}$ y su homólogo de $\mathrm{Cu}\left|\mathrm{Cu}^{2+}\right| \mathrm{Cu}_{2} \mathrm{SO}_{4^{\prime}}, 1 \mathrm{M}|| \mathrm{W}^{0}$ al $95 \%$ y $99 \%$ de confianza.

Tabla 7. Resultados de la evaluación de la significancia de correlación para los sistemas electroquímicos 1 y 2 .

\begin{tabular}{cccc}
\hline Día & Sistema 1 & Sistema 2 & t crítico \\
\cline { 2 - 4 } & $\mathbf{t}$ & $\mathbf{t}$ & $\mathbf{9 5 \%}$ \\
\hline 1 & $22,338^{\text {ns }}$ & $18,230^{\text {ns }}$ & 12.71 \\
2 & $31,607^{\text {ns }}$ & $14,107^{\text {ns }}$ & \\
3 & $10,493^{*}$ & $14,107^{\text {ns }}$ & \\
4 & $7,604^{*}$ & $15,780^{\text {ns }}$ & \\
\hline
\end{tabular}

El sistema electroquímico 2 presenta una correlación significativa al $95 \%$ de confianza durante los 4 días de evaluación, mientras que en el sistema electroquímico 1 se observa una acusada relación lineal significativa al $95 \%$ de confianza durante los 2 primeros días de evaluación. Cabe anotar que ambos sistemas electroquímicos se mantuvieron durante los 4 días de evaluación, sin cambio de soluciones internas y conservadas en sulfato de cobre $1 \mathrm{M}$ y agua como líquido de almacenamiento, respectivamente.

Tabla 8. Potencial eléctrico (eV) en función del tiempo para soluciones tampón de pH 4.01, pH 7.00 y pH 9.21.

\begin{tabular}{ccccccc}
\hline Tiempo & \multicolumn{3}{c}{ Sistema 1, eV, V } & \multicolumn{3}{c}{ Sistema 2, eV, V } \\
\cline { 2 - 7 } $\mathbf{0 - 9 0 0 ~ s H}$ & $\mathbf{p H}$ & $\mathbf{p H}$ & $\mathbf{p H}$ & $\mathbf{p H}$ & $\mathbf{p H}$ & $\mathbf{p H}$ \\
& $\mathbf{4 . 0 1}$ & $\mathbf{7 . 0 0}$ & $\mathbf{9 . 2 1}$ & $\mathbf{4 . 0 1}$ & $\mathbf{7 . 0 0}$ & $\mathbf{9 . 2 1}$ \\
\hline Media & $-0,045$ & $-0,177$ & $-0,352$ & $-0,105$ & $-0,242$ & $-0,417$ \\
Des.Est. & 0,007 & 0,005 & 0,002 & 0,003 & 0,002 & 0,007 \\
Rango & 0,026 & 0,022 & 0,011 & 0,012 & 0,008 & 0,023 \\
\hline
\end{tabular}


Difieren desde el punto de vista de estabilidad en función del $\mathrm{pH}$, siendo el sistema 1 más estable a rangos de $\mathrm{pH}$ alto y el sistema 2 inversamente, a pH bajo, lo cual demuestra la influencia de la solución interna del microelectrodo de referencia.

Con respecto a la estabilidad en función del tiempo; el microelectrodo cuya solución interna es de $\mathrm{CuSO}_{4^{\prime}}$ a partir del tercer día el cobre se corroe por la presencia de la solución de carácter ácido; a partir del cuarto día se observan fenómenos de cristalización en el tapón de algodón; análogamente en el microelectrodo con solución interna de agua desmineralizada, a partir del tercer día es notable la presencia de burbujas a causa de la pérdida de líquido.

Estos fenómenos influyen en el desempeño práctico y se demuestran en la tabla 4 , en donde durante los tres primeros días se mantiene una alta sensibilidad en función del $\mathrm{pH}$, la cual comienza a decrecer considerablemente a partir del cuarto día en ambos sistemas; de acuerdo a la tabla 5 en el análisis de significancia de correlación, en el sistema 1 es más acusada la relación lineal durante los dos primeros días de estudio que en el sistema 2 al obtenerse valores más altos de t, sin embargo, la disminución de la correlación se encuentra vinculada a la pérdida de su solución interna y en el sistema 2, a pesar de los fenómenos de corrosión observados se mantiene la significancia lineal entre su respuesta en función del $\mathrm{pH}$.

De acuerdo a los resultados experimentales, los microelectrodos de referencia deben ser regenerados al segundo día de ensayo como plazo máximo, en especial el electrodo de referencia del sistema 2 incluyendo el reemplazo de su alambre de cobre y de la elaboración de una solución de $\mathrm{CuSO}_{4} 1 \mathrm{M}$. En cambio, el sistema 1 únicamente requiere substituir el tapón de algodón y su solución interna de agua desmineralizada, acompañado de un lijado del contacto de conexión a tierra del multímetro, además se evita correr el riesgo de contaminar la muestra debido a la naturaleza semipermeable del microelectrodo. Por lo que se decidió utilizar el sistema 1.

\subsection{Estudio estadístico}

En la Tabla 9 se presentan los resultados del Análisis de Varianza de dos factores para el análisis de Precisión a cinco niveles de concentración:

Tabla 9. Análisis de Varianza para la evaluación de precisión

\begin{tabular}{|c|c|c|c|c|c|c|c|}
\hline $\begin{array}{c}\text { \% Ác. } \\
\text { Cítrico(p/v) }\end{array}$ & $\begin{array}{c}\text { Origen de las } \\
\text { variaciones }\end{array}$ & GL & $\begin{array}{l}\text { Suma de } \\
\text { cuadrados }\end{array}$ & Cuadrado medio & $\mathbf{F}$ & $\begin{array}{l}\mathrm{F} \mathrm{tab} \\
95 \%\end{array}$ & $\begin{array}{l}\mathrm{F} \text { tab } \\
99 \%\end{array}$ \\
\hline \multirow[t]{4}{*}{$0.10 \%$} & Repeticiones & 9 & $8,56 \mathrm{E}-05$ & 9,51E-06 & $0,60^{\text {ns }}$ & 2,46 & 3,60 \\
\hline & Días & 2 & 5,56E-05 & 2,78E-05 & $1,75^{\text {ns }}$ & 3,55 & 6,01 \\
\hline & Error & 18 & 2,87E-04 & $1,59 \mathrm{E}-05$ & & & \\
\hline & Total & 29 & $4,28 \mathrm{E}-04$ & & & & \\
\hline \multirow[t]{4}{*}{$0.50 \%$} & Repeticiones & 9 & $8,65 \mathrm{E}-04$ & 9,61E-05 & $0,91^{\text {ns }}$ & & \\
\hline & Días & 2 & 2,87E-04 & $1,43 E-04$ & $1,36^{\mathrm{ns}}$ & & \\
\hline & Error & 18 & 1,90E-03 & $1,05 \mathrm{E}-04$ & & & \\
\hline & Total & 29 & $3,05 \mathrm{E}-03$ & & & & \\
\hline \multirow[t]{4}{*}{$1.00 \%$} & Repeticiones & 9 & $7,36 \mathrm{E}-04$ & $8,18 \mathrm{E}-05$ & $0,59^{\text {ns }}$ & & \\
\hline & Días & 2 & 1,17E-03 & $5,84 \mathrm{E}-04$ & $4,19^{*}$ & & \\
\hline & Error & 18 & 2,51E-03 & $1,40 \mathrm{E}-04$ & & & \\
\hline & Total & 29 & 4,42E-03 & & & & \\
\hline \multirow[t]{4}{*}{$5.00 \%$} & Repeticiones & 9 & $1,71 \mathrm{E}-01$ & 1,90E-02 & $1,27^{\text {ns }}$ & & \\
\hline & Días & 2 & $2,05 \mathrm{E}-02$ & 1,03E-02 & $0,68^{\text {ns }}$ & & \\
\hline & Error & 18 & $2,71 \mathrm{E}-01$ & $1,50 \mathrm{E}-02$ & & & \\
\hline & Total & 29 & 4,62E-01 & & & & \\
\hline \multirow[t]{4}{*}{$10.00 \%$} & Repeticiones & 9 & $1,37 \mathrm{E}-01$ & $1,52 \mathrm{E}-02$ & $0,95^{\text {ns }}$ & & \\
\hline & Días & 2 & 1,71E-03 & $8,56 \mathrm{E}-04$ & $0,05^{\text {ns }}$ & & \\
\hline & Error & 18 & $2,89 \mathrm{E}-01$ & 1,61E-02 & & & \\
\hline & Total & 29 & 4,28E-01 & & & & \\
\hline
\end{tabular}


El efecto de las repeticiones no es significativo al $95 \%$ y $99 \%$ en los cinco niveles de análisis, sin embargo, el efecto del tiempo de análisis es significativo al $95 \%$ para el nivel de análisis al $1 \%(\mathrm{p} / \mathrm{v})$ debido a que para este nivel se utilizó una cantidad de titulante superior al valor nominal de la microbureta de bajo costo $(1.00 \mathrm{~mL})$, por lo que la incertidumbre asociada al método es mayor a este nivel. Considerando este factor se decidió realizar para los siguientes niveles de análisis $(5 \%$ y $10 \%)$ diluciones en agua desmineralizada descarbonatada.

Tabla 10. Resultados parámetros estadísticos

\begin{tabular}{lc}
\hline \multicolumn{1}{c}{ Parámetro } & Resultado \\
\hline Límite de detección & $0,02 \%$ Ác. Cítrico $(\mathrm{p} / \mathrm{v})$ \\
Límite de cuantificación & $0.03 \%$ Ác. Cítrico $(\mathrm{p} / \mathrm{v})$ \\
Exactitud, CV & $1.15 \%-6.54 \%$ \\
Exactitud, Error Absoluto & $-0.05 \%-0.00 \% \mathrm{AC}(\mathrm{p} / \mathrm{v})$ \\
Exactitud, Error Relativo & $0.00 \%-0.91 \%$ \\
Sensibilidad, Recuperación & $100 \%-107 \%$ \\
\hline
\end{tabular}

El límite de cuantificación obtenido $10.03 \%$-p/v- Acido cítricol permite cuantificar por este método la acidez valorable presente en la mayoría de muestras de jugos, frutas y bebidas tipo jugo comerciales, ya que el rango promedio en estas matrices se encuentra entre el $0.1 \%$ y el $10 \%(\mathrm{p} / \mathrm{v})$ de ácido cítrico. Los valores referentes a la exactitud obtenidos por medio del ensayo de recuperación cuyo valor estándar es referido al obtenido por el método de la AOAC y el evaluado en este estudio, nos permitió demostrar que el método de bajo costo no posee errores sistemáticos de consideración, errores relativos correspondientes a $0.00 \%$ a $0.91 \%$ son considerados aceptables. Se obtuvieron porcentajes de recuperación de 100\% y 107\% para los 5 niveles evaluados, lo cual se considera aceptable; sin embargo, es necesario evaluar con más detalle este factor a mayor nivel de concentración con la finalidad de que los errores aleatorios no afecten considerablemente a los resultados obtenidos por los pequeños incrementos de analito.

\subsection{Estudio comparativo}
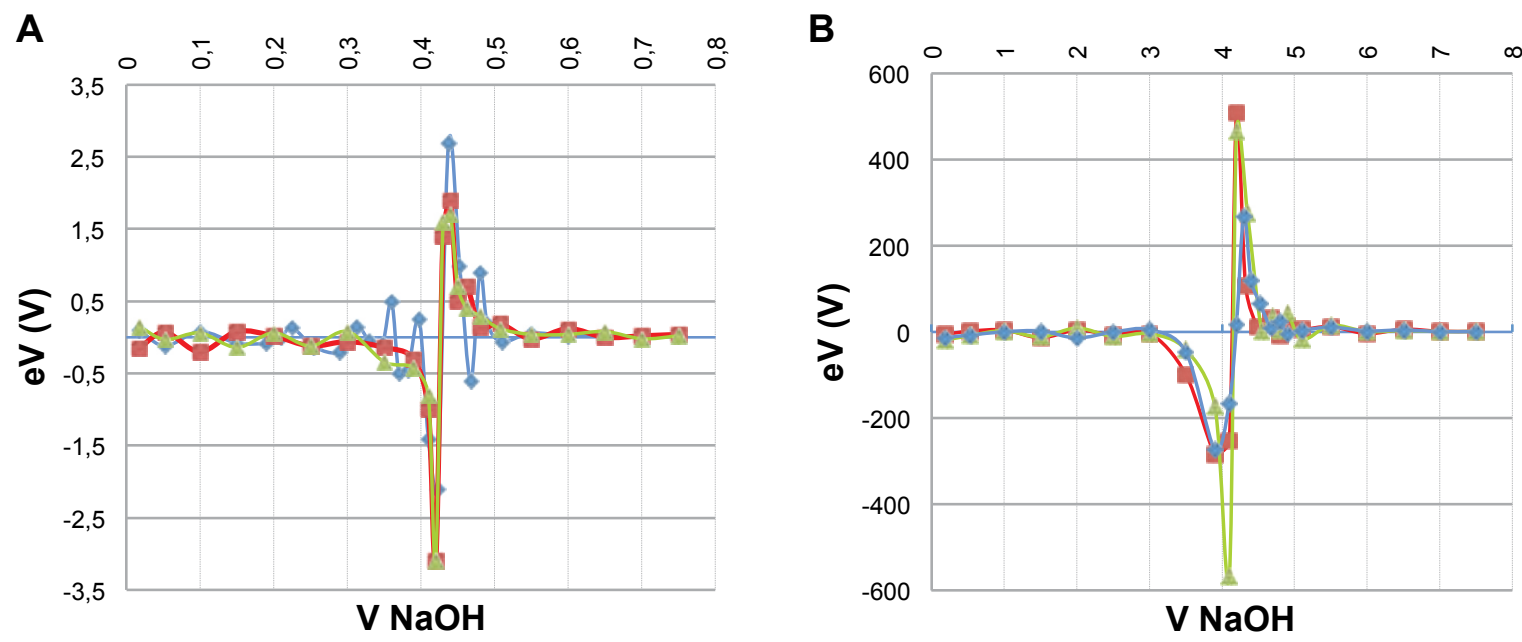

Figura 4. Muestra $A 1$, segunda derivada ( $\left.d^{\prime \prime} \mathrm{eV} / \mathrm{d}^{\prime \prime} \mathrm{V}\right)$ en función de volumen de $\mathrm{NaOH} 0.1003 \mathrm{~N}(\mathrm{~mL})$, A: Método micropotenciométrico de bajo costo, B: Método potenciométrico clásico. 
Tabla 11. Medias de los resultados del análisis de las muestras de bebidas (bebida tipo jugo, frutas, néctar) entre el método potenciométrico $P(x)$ y el método micropotenciométrico de bajo costo $M P(y)$..

\begin{tabular}{ccc|ccc|ccc}
\hline \multicolumn{1}{c}{ Resultado, \% Ácido Cítrico $(\% \mathbf{p} / \mathbf{v})$} & \multicolumn{1}{c}{ M } \\
\hline Muestra & MP & $\mathbf{P}$ & Muestra & MP & $\mathbf{P}$ & Muestra & MP & P \\
\hline A1 & 0,28 & 0,27 & B1 & 5,22 & 5,05 & C1 & 0,29 & 0,27 \\
A2 & 0,31 & 0,30 & B2 & 0,92 & 0,91 & C2 & 0,28 & 0,28 \\
A3 & 0,45 & 0,42 & B3 & 3,93 & 3,79 & C3 & 0,59 & 0,57 \\
A4 & 0,46 & 0,45 & B4 & 8,04 & 7,60 & C4 & 0,28 & 0,27 \\
A5 & 0,31 & 0,29 & B5 & 1,58 & 1,40 & C5 & 0,21 & 0,21 \\
A6 & 0,11 & 0,11 & B6 & 2,24 & 2,27 & C6 & 0,16 & 0,16 \\
A7 & 0,30 & 0,29 & B7 & 0,16 & 0,19 & C7 & 0,40 & 0,38 \\
A8 & 0,26 & 0,26 & B8 & 0,56 & 0,58 & C8 & 0,27 & 0,26 \\
A9 & 0,34 & 0,32 & B9 & 2,11 & 1,90 & C9 & 0,26 & 0,24 \\
A10 & 0,46 & 0,43 & B10 & 0,21 & 0,20 & C10 & 0,20 & 0,19 \\
\hline
\end{tabular}

Figura 5. Recta de regresión para la comparación del método potenciométrico $P(x)$ y el método micropotenciométrico de bajo costo MP (y).

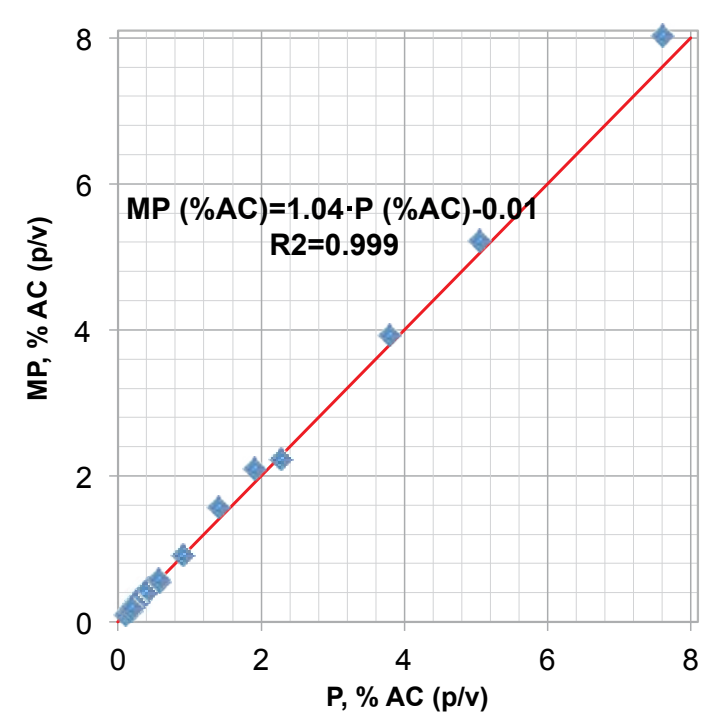

Tabla 12. Resultados de la evaluación de significancia entre el método potenciométrico y el método micropotenciométrico de bajo costo.

\begin{tabular}{lcc}
\hline & MP & P \\
\hline Varianza & 3,113 & 2,823 \\
Observaciones & 30 & 30 \\
Grados de libertad & 29 & \\
Estadístico t & 2,531 & \\
Valor crítico de $t$ (dos colas), 99\% & 2,756 & \\
Valor crítico de t (dos colas), 95\% & 2,045 & \\
\hline
\end{tabular}

Por medio del método de la recta de regresión, se obtuvo una alta correlación $\left(r^{2}=0.999\right)$ y las desviaciones de la pendiente (1.04) y de la ordenada al origen $(-0.01)$ se aproximan al comportamiento "ideal" (pendiente $=1$, ordenada $=0, r=1$ ); de acuerdo al contraste t de significancia entre la media de los resultados obtenidos por cada sistema, entre los métodos no existe diferencia significativa al $99 \%$ de confianza pero sí es significativo ligeramente al $95 \%$ de confianza. Esta situación puede explicarse debido al amplio intervalo de concentraciones en el cual se trabajó, motivo por el cual el contraste t es cuestionado por muchos químicos analíticos ya que tal comparación es óptima cuando el intervalo de concentración es muy limitado. Sin embargo, por la alta correlación obtenida y por que el método no es significativo al $99 \%$ de confianza se puede aseverar que no existe diferencia estadística para la obtención de resultados de acidez valorable por el método micropotenciométrico de bajo costo y el método potenciométrico con electrodo de vidrio.

\section{Conclusiones}

Se construyeron dos sistemas microelectroquímicos $\left(\mathrm{Cu}\left|\mathrm{Cu}^{2+}\right| \mathrm{H}_{2} \mathrm{O}|| \mathrm{W}^{0}\right.$ y $\mathrm{Cu}\left|\mathrm{Cu}^{2+}\right| \mathrm{Cu}_{2} \mathrm{SO}_{4^{\prime}}, \mathrm{MM}|| \mathrm{W}^{0} \mid$ con materiales de bajo costo, fácil adquisición, fabricación y regeneración sencilla.

Ambos sistemas ofrecen marcadas relaciones lineales de su respuesta frente el $\mathrm{pH}$, con elevados coeficientes de correlación, siendo el sistema $\mathrm{Cu}\left|\mathrm{Cu}^{2+}\right| \mathrm{H}_{2} \mathrm{O}|| \mathrm{W}^{0}$ 
más estable a $\mathrm{pH}$ alto e inversamente, el sistema $\mathrm{Cu}\left|\mathrm{Cu}^{2+}\right| \mathrm{Cu}_{2} \mathrm{SO}_{4}, 1 \mathrm{M}|| \mathrm{W}^{0}$ a pH bajo.

A pesar de que ambos sistemas microelectroquímicos ofrecen resultados estadísticamente similares, se seleccionó al sistema $\mathrm{Cu}\left|\mathrm{Cu}^{2+}\right| \mathrm{H}_{2} \mathrm{O}|| \mathrm{W}^{0}$ por su elevado desempeño, mayor facilidad de montaje y regeneración, menor riesgo de operación y costo económico que su homólogo de $\mathrm{Cu}\left|\mathrm{Cu}^{2+}\right| \mathrm{Cu}_{2} \mathrm{SO}_{4^{\prime}}, 1 \mathrm{M}|| \mathrm{W}^{0}$, cumpliendo todos los requisitos mínimos para un electrodo de referencia.

Con un volumen de $1.00 \mathrm{~mL}$ de muestra se obtuvo una baja dispersión de resultados, motivo por el cual se trabajó con esta cantidad durante todo el estudio como medida aproximada adecuada para el método micropotenciométrico de acidez valorable.

El límite de detección para el método micropotenciométrico para la determinación de acidez valorable se estimó en $0.02 \%$ de ácido cítrico $(\mathrm{p} / \mathrm{v})$ y el límite de cuantificación corresponde a $0.03 \%$ de ácido cítrico $(\mathrm{p} / \mathrm{v})$.
El método micropotenciométrico es altamente preciso $y$ exacto en un amplio rango de concentraciones de ácido cítrico, utilizado como analito diana.

El método micropotenciométrico es capaz de diferenciar entre pequeños incrementos de concentración de ácido cítrico con porcentajes de recuperación cercanos al $100 \%$.

Los resultados obtenidos por el método micropotenciométrico de bajo costo tuvieron concordancia con el obtenido por el método potenciométrico clásico con electrodo de vidrio, ambos resultaron ser comparables por el método de la recta de regresión y por el estadístico $t$ al $99 \%$ de confianza.

El método analítico propuesto para la determinación de acidez valorable demostró su aplicabilidad en jugos naturales, bebidas tipo jugo y néctares/zumos con ventajas económicas, operativas, de portabilidad y ambientales frente a su homólogo clásico con electrodo de vidrio.

\section{Referencias}

1. Nielsen, S. (2009). Análisis de los Alimentos. Zaragoza: Acribia.

2. Baeza, A. (2003). Titulaciones Ácido-Base Potenciométricas a Microescala Total con Microsensores de pH y de Referencia de bajo costo. Rev. Chil. Educ. Cient. , 1 (2).

3. Skoog, D., \& Holler, F. N. (2001). Principios de Análisis Instrumental (Vol. 5ta edición). México: McGraw Hill.

4. Liu, C., Bocchicchio, B., \& Overmyer, P. (1980). A Palladium-Palladium Oxide Miniature pH Electrode. Science, 207 (188-189).

5. Smirnova, O., \& Mikhailova, A. (2000). Potentiometric Parameters of a PVC-NaV6O15-Graphite Electrode. Russ. J. Elec. , 36 (8).

6. Arnold, M., \& Meyerhoff, M. (1984). Ion-Selective Electrodes. Anal. Chem. , 56 (5).

7. Kurzweil, P. (2009). Metal Oxides and lon-Exchanfing Surfaces as pH Sensors in Liquids: State-of-the-Art and Outlook. Sensors , 9 (49554985).

8. Galwa, N., \& El Nahhal, I. (2007). Development of a Novel Solid-State pH Sensor Electrode Based on Titanium Oxide Thin Film as a Indicator Electrode in Potentiometric Acid-Base Titrations in Fused NaNO3 at 350 ${ }^{\circ} \mathrm{C}$. J. Disper. Sci. Tech. 28 (757-764).

9. García-González, D., \& Aparicio, R. (2002). Sensors: From Biosensors to the Electronic Nose. Grasas y Aceites, 53 (2).

10. Hughes, R., Ricco, A., Butler, M., \& Martin, S. (1991). Chemical Microsensors. Science , 254 (74-79).

11. Aytaç, A., Kabasakalo lu, M., Sari, B., \& Talu, M. (2004). Ion-Selective Electrodes Prepared with Polyaniline Membranes. Russ. J. of Elec., 40 (7).

12. Sotomayor, P., Raimundo, I., Zarbin, A., Rohwedder, J., Oliveira, G., \& Alves, O. (2001). Construction and evaluation of an optical pH sensor based on polyaniline-porous Vycor glass nanocomposite. Sens. Act. B , 74 (157-162).

13. Caldwell, P. (1954). An Investigation of the Intracellular pH of Crab Muscle Fibres by Means of Micro-Glass and Micro-Tungsten Electrodes. J. Physiol. , 126 (169-180).

14. Dimitrakopoulos, L., Dimitrakopoulos, T., Alexander, P., Logic, D., \& Hibbert, B. (1998). A Tungsten Oxide Coated Wire Electrode Used as a pH Sensor in Flow Injection Potentiometry. Anal. Commun., 35 (395-398).

15. Baeza, A. (2003). Microbureta a Microescala Total para Titulometría. Rev. Chil. Educ. Cient. , 1 (2).

16. Vargas, J., Baeza, A. U., Rodríguez, J., \& Cáceres, L. (2004). Titulaciones Ácido-Base a Microescala Química utilizando Microsensores de $\mathrm{pH}$ y Microelectrodo de referencia: Adquisición de datos con nuevas tecnologías. Rev. Chil. Educ. Cient. , 2 (2).

17. Box, G., \& Hunter, W. H. Estadística para Investigadores. México: Reverté.

18. Miller, J., \& Miller, J. (2002). Estadística y Quimiometría para Química Analítica. Madrid: Prentice Hall.

19. EURACHEM Group. (1998). The Fitness for Purpose of Analytical Methods. Middlesex: Eurachem Working Group.

20. Angelini, N. (2005). Validación de Métodos Químicos Desarrollados en el Laboratorio. Buenos Aires: FAO. 